\title{
Conducting polyaniline/reduced graphene oxide-modified carbon paste electrode for the electrochemical multi- detection of ascorbic acid and uric acid
}

\author{
Janine Micah G. Baricaua ${ }^{1}$, Jolleen Natalie I. Balitaan ${ }^{1}$, Karen S. Santiago $^{1,2}$ \\ ${ }^{1}$ Department of Chemistry, College of Science, University of Santo Tomas, España Blvd., Manila \\ 1015, Philippines, \\ ${ }^{2}$ Research Center for the Natural and Applied Sciences, University of Santo Tomas, España BIvd., \\ Manila 1015, Philippines \\ Corresponding author's e-mail address kssantiago@ust.edu.ph
}

\begin{abstract}
:
This study presents a simultaneous electrochemical detection of ascorbic acid (AA) and uric acid (UA) using fabricated polyaniline/reduced graphene oxide-modified carbon paste electrode (PANI/rGO$\mathrm{mCPE})$. PANI/rGO was synthesized via in situ chemical polymerization route, and characterized using FTIR, SEM, and 4-point conductivity probe. The sensor's performance was evaluated towards standard solutions of AA and UA. FTIR and conductivity studies proved that PANI/rGO composite was successfully synthesized. The FTIR spectrum of PANI/rGO shows the characteristic peaks of PANI: $3300-3500 \mathrm{~cm}-1$ (amine N-H bonds); 1400-1750 (aromatic rings). Combining PANI and rGO provided enhanced conductivity and surface area as compared to pristine PANI and rGO. Voltammetric studies showed direct proportional relationship between anodic peak current against analyte concentration. Based on voltammetric studies, the redox capability of synthesized electrodes was found to show an increasing trend of CPE < PANI-mCPE < PANI/rGO-mCPE. Among the three tested electrodes, the fabricated PANI/rGO-mCPE exhibited the best performance for the multi-detection of AA and UA.
\end{abstract}

Key words: polyaniline, reduced graphene oxide, multi-detection, voltammetry

\section{Introduction}

Ascorbic acid and uric acid play important roles in metabolism systems [1]; thus, concentrations of these acids may serve as biomarkers of physiological conditions. AA level, for instance, is related to oxidative stress [2], while extreme abnormal concentrations of UA signal possible gout or hyperuricemia [3]. High-performance liquid chromatography (HPLC), which employs different detectors, is commonly used in simultaneous AA and UA determination. However, the instrument involved is expensive, and requires trained personnel to operate. Also, this technique suffers from low sensitivity and selectivity [3-5]. With these aforementioned considerations, there is still a high interest in developing low-cost devices that operate in facile and fast way, and that can measure 2 or more target analytes in a single sensor exposure. This particular study was, hence, geared towards the construction of polyaniline/reduced graphene oxide-modified carbon paste electrode for the multi-detection of ascorbic acid and uric acid.

\section{Methodology}

Reduced graphene oxide was prepared using Hummer's method [6]. $\mathrm{H}_{2} \mathrm{SO}_{4}(98 \%, 120 \mathrm{~mL})$, graphite $(3.0 \mathrm{~g})$, and $\mathrm{NaNO}_{3}(1.5 \mathrm{~g})$ were mixed, and the resulting mixture was stirred for $2 \mathrm{~h}$ in an ice bath. After stirring, $\mathrm{KMnO}_{4}(18 \mathrm{~g})$ was added slowly. The solution was stirred for $18 \mathrm{~h}$ in a $\mathrm{H}_{2} \mathrm{O}$ bath, which resulted to a dark green solution. Cold distilled $\mathrm{H}_{2} \mathrm{O}(850 \mathrm{~mL})$ was added and stirred for $30 \mathrm{~min}$. Excess $\mathrm{KMnO}_{4}$ was eliminated from the solution by adding $3 \mathrm{~mL}$ of $\mathrm{H}_{2} \mathrm{O}_{2}$ dropwise under stirring for $2 \mathrm{~h}$. The solution was filtered, and washed with $\mathrm{HCl}$, deionized $\mathrm{H}_{2} \mathrm{O}$, and ethanol. It was centrifuged and the supernatant was subjected to osmosis, then the GO solution was freeze-dried. PANI/rGO was prepared via in situ chemical oxidative polymerization. Aniline:GO (1:5), and $1 \mathrm{M} \mathrm{HCl}$ were added to double distilled $\mathrm{H}_{2} \mathrm{O}$. This mixture was stirred for $30 \mathrm{~min}$ at RT. 
$\left(\mathrm{NH}_{4}\right)_{2} \mathrm{~S}_{2} \mathrm{O}_{8}$ was added to the resulting mixture with continuous stirring for $4 \mathrm{~h}$. Synthesized materials were characterized using Fourier Transform Infrared Spectroscopy (FTIR) Shimadzu IR Prestige-21, Scanning Electron Microscope (SEM) Hitachi TM 3000, and an improvised 4-point probe. The substrate was prepared using a $\mathrm{Cu}$ wire attached to a circular $\mathrm{Cu}$ plate, and inserted in a cylindrical high density PET tube. Graphite powder was mixed with mineral oil using agate mortar and pestle. Then PANI/rGO was mixed with graphite/oil. This PANI/rGO/oil mixture was filled inside the tube and was pressed until a smooth surface was obtained. Through cyclic voltammetry (CV), the performance of PANI/rGO-mCPE (working electrode) was investigated by monitoring the anodic peaks of increasing concentrations of $\mathrm{AA}$ and UA (2-8 mM analyte, 0.1 M phosphate buffer at $\mathrm{pH}=7$ ). The PANI/-mCPE was used as the working electrode, against $\mathrm{Ag} / \mathrm{AgCl} \mathrm{RE}$ and $\mathrm{Pt}$ auxiliary electrode. CV studies was conducted at a sweeping potential range of -0.2 to $1.0 \mathrm{~V}$ and a scan rate of $5 \mathrm{mVs}^{-1}$ for 3 cycles.

\section{Results and discussion}

FTIR spectral studies showed the characteristic peaks of PANI/rGO, and GO. The peak at $3400 \mathrm{~cm}^{-1}$ of PANI/rGO was linked to $\mathrm{N}$ $\mathrm{H}$ stretching mode. A peak corresponding to $\mathrm{C}$ $\mathrm{H}$ plane bending on benzenoid ring was seen at $3100 \mathrm{~cm}^{-1}$ for PANI/rGO. The 1750 and 1690 $\mathrm{cm}^{-1}$ peaks for $\mathrm{PANI} / \mathrm{rGO}$ contribute to the $\mathrm{C}=\mathrm{N}$ stretching mode for imine. $\mathrm{C}=\mathrm{C}$ stretching vibration for quinoid and benzenoid rings was seen at 1600 and $1400 \mathrm{~cm}^{-1}$, respectively. C-N stretching mode for benzenoid ring is seen at $1150 \mathrm{~cm}^{-1}$. In the fingerprint region, $\mathrm{C}-\mathrm{H}$ plane bending vibration formed during protonation can be seen at $700 \mathrm{~cm}^{-1}$ for PANI/rGO. It can be seen that the strong and broad absorption peak at $3200 \mathrm{~cm}^{-1}$ corresponds to alcohol. $\mathrm{CO}_{2}$ corresponds to the peak at $2360 \mathrm{~cm}^{-1}$. The $\mathrm{C}=\mathrm{O}$ stretching vibration was attributed at $1700 \mathrm{~cm}^{-1}$ and $\mathrm{C}=\mathrm{C}$ plane vibration is at $1600 \mathrm{~cm}^{-1}$. Via cyclic voltammetric analyses, 2 anodic peaks were seen which correspond to mixed solutions of $A A$, and UA. The first anodic peak at the potential range of 0.1 to $0.3 \mathrm{~V}$ corresponds to ascorbic acid while the second anodic peak at 0.4 to $0.6 \mathrm{~V}$ confirms the presence of uric acid. SEM morphological analyses revealed that PANI has a porous, spongy-like structure which indicates high surface area, while rGO exhibits a wrinkled structure with aggregated sheets. When PANI and rGO are incorporated together, enhanced surface area was observed. Furthermore, conductivity increased by nearly nine-hundred folds, i.e., $2.17 \times 10^{-5} \mathrm{~S} \mathrm{~cm}^{-1}$ for
PANI and $2.16 \times 10^{-4} \mathrm{~S} \mathrm{~cm}^{-1}$ for PANI/rGO. As probed via cyclic voltammetric analyses, the redox capability of synthesized electrodes was found to show an increasing trend of CPE < PANI-mCPE <PANI/rGO-mCPE. The figures of merit of the fabricated PANI/rGO-mCPE, when exposed to mixed solutions of $A A$ and UA, are summarized in Tab. 1 .

Tab. 1: Summary of sensors' performance towards multi-detected $A A$ and UA $(n=3)$.

\begin{tabular}{|l|c|c|}
\hline \multicolumn{1}{|c|}{ Figures of merits } & $\begin{array}{c}\text { PANI/rGO-mCPE } \\
\text { exposed to AA }\end{array}$ & $\begin{array}{c}\text { PAN } 1 / \text { rGO- } \\
\text { mCPE exposed } \\
\text { to UA }\end{array}$ \\
\hline Sensitivity $(\mathrm{m}), \mu \mathrm{A} / \mathrm{mM}$ & 16.67 & 9.490 \\
\hline Linearity $(r)$ & 0.9977 & 0.9826 \\
\hline Linear working range, $\mathrm{mM}$ & $2-8$ & $2-8$ \\
\hline Limit of detection, mM & 1 & 1 \\
\hline Response time, $\mathrm{s}$ & 59 & 115 \\
\hline Repeatability, \%RSD & 3.63 & 7.21 \\
\hline
\end{tabular}

\section{Conclusion}

Combining PANI and rGO, through in situ chemical oxidative polymerization, resulted to higher electrical conductivity, and increased surface area, which effectively enhanced the performance of the sensor in the simultaneous detection of ascorbic acid and uric acid.

\section{References}

[1] N.F. Atta, M.F. El-Kady, A. Galal, Simultaneous determination of catecholamines, uric acid and ascorbic acid at physiological levels using poly $(\mathrm{N}$ methylpyrrole)/Pd-nanoclusters sensor, Analytical Biochemistry 400 (2010) 78-88.

[2] J. Lykkesfeldt, Cancer Epidemiol., Biomarkers Prev. 16 (2007) 2513.

[3] X. Li, A.A. Franke, Fast HPLC-ECD analysis of ascorbic acid, dehydroascorbic acid and uric acid, Journal of Chromatography B, 877 (2009) 853-856.

[4] R. Zuo, S. Zhou, Y. Zuo, Y. Deng, Determination of creatinine, uric and ascorbic acid in bovine milk and orange juice by hydrophilic interaction HPLC, Food Chemistry 182 (2015) 242-245.

[5] R. Kand'ár, P. Drábkováa, R. Hampl, The determination of ascorbic acid and uric acid in human seminal plasma using an HPLC with UV detection, Journal of Chromatography B, 879 (2011) 2834- 2839.

[6] K. Narasimharao, G. Venkata Ramana, D. Sreedhar and V. Vasudevarao, Synthesis of Graphene Oxide by Modified Hummers Method and Hydrothermal Synthesis of Graphene-NiO Nano Composite for Supercapacitor Application, J Material Sci Eng (2016) 5:6. 